\title{
Gut microbial biomarkers for the treatment response in first- episode, drug-naïve schizophrenia: a 24-week follow-up study
}

\author{
Xiuxia Yuan ${ }^{1,2,3,11}$, Yunpeng Wang ${ }^{4,11}$, Xue Li ${ }^{1,2,3,11}$, Jiajun Jiang ${ }^{5}$, Yulin Kang ${ }^{6}$, Lijuan Pang ${ }^{1,2,3}$, Peifen Zhang ${ }^{5}$, Ang Li ${ }^{7}$, Luxian Lv ${ }^{8}$,
} Ole A. Andreassen $\mathbb{1 0}^{9}$, Xiaoduo Fan ${ }^{10}$, Shaohua $\mathrm{Hu}^{5 凶}$ and Xueqin Song $\mathbb{1}^{1,2,3 凶}$

(c) The Author(s) 2021

Preclinical studies have shown that the gut microbiota can play a role in schizophrenia (SCH) pathogenesis via the gut-brain axis. However, its role in the antipsychotic treatment response is unclear. Here, we present a 24-week follow-up study to identify gut microbial biomarkers for SCH diagnosis and treatment response, using a sample of 107 first-episode, drug-naïve SCH patients, and 107 healthy controls (HCs). We collected biological samples at baseline (all participants) and follow-up time points after risperidone treatment (SCH patients). Treatment response was assessed using the Positive and Negative Symptoms Scale total (PANSS-T) score. False discovery rate was used to correct for multiple testing. We found that SCH patients showed lower a-diversity (the Shannon and Simpson's indices) compared to HCs at baseline $\left(p=1.21 \times 10^{-9}, 1.23 \times 10^{-8}\right.$, respectively). We also found a significant difference in $\beta$-diversity between SCH patients and HCs $(p=0.001)$. At baseline, using microbes that showed different abundance between patients and controls as predictors, a prediction model can distinguish patients from HCs with an area under the curve (AUC) of 0.867 . In SCH patients, after 24 weeks of risperidone treatment, we observed an increase of a-diversity toward the basal level of HCs. At the genus level, we observed decreased abundance of Lachnoclostridium $(p=0.019)$ and increased abundance Romboutsia $(p=0.067)$. Moreover, the treatment response in $\mathrm{SCH}$ patients was significantly associated with the basal levels of Lachnoclostridium and Romboutsia ( $p=0.005$ and 0.006 , respectively). Our results suggest that SCH patients may present characteristic microbiota, and certain microbiota biomarkers may predict treatment response in this patient population.

Translational Psychiatry (2021)11:422; https://doi.org/10.1038/s41398-021-01531-3

\section{INTRODUCTION}

Schizophrenia $(\mathrm{SCH})$ is a chronic and severe mental disorder, characterized by impaired cognitive functions and social disability [1]. Despite that SCH incurs a heavy burden on family and society, the etiology of the disease is largely unknown $[2,3]$. Preclinical research has suggested that the dysbiosis of gut microbiota could perturb several pathophysiological pathways thus contributing to the risk of $\mathrm{SCH}$, e.g., disruption of glutamatergic neurotransmission and the glutamate-glutamine-GABA ( $\gamma$-aminobutyric acid) cycle $[4,5]$, and dysregulation of tryptophan's metabolites and neuroimmune activation [6-8]. It was also suggested that the immune and inflammatory mechanisms may be the link between the gut microbiota and the risk of $\mathrm{SCH}$ [9]. The SCH-enriched bacteria recipient mice showed deficits in social behaviors and changes in the expression of immune/inflammation-related genes in the intestine, suggesting that dysfunction of the gut microbiota may affect the functioning of inflammation pathways and schizophrenia-like behavior in the animals [10]. However, the clinical implications of these preclinical findings are still unclear.
The associations between the gut microbial compositions and the risk of $\mathrm{SCH}$ have also been reported in several psychiatric patient studies [5, 9-13]. The alteration of phylum especially Firmicutes was reported to be associated with psychiatric disorders, e.g., schizophrenia [11, 12, 14], depression [15, 16], and autism [17], although specific findings vary across different studies. Oropharyngeal microbiome analysis has shown that $\mathrm{SCH}$ patients had a higher abundance of Firmicutes but the lower abundance of Bacteroidetes and Actinobacteria compared to healthy controls (HCs) [11]; intestinal microbiome analysis has found a higher abundance of phylum Proteobacteria [12] and Actinobacteria $[9,14]$ but the lower abundance of Firmicutes $[12,14]$ in SCH patients compared to HCs. Genus analysis has shown that some genera from Firmicutes showed lower levels in SCH patients than in HCs, such as Blautia, Coprococcus, Roseburia, Blautia, Streptococcus, Enterococcus [12, 13]; other genera from Firmicutes, including Megasphaera, Lactobacillus, and Clostridium, were enriched in $\mathrm{SCH}[12,13]$. In addition, several genera from the phyla Proteobacteria [12] (e.g., Succinivibrio, and Klebsiella) and

\footnotetext{
'Department of Psychiatry, The First Affiliated Hospital/Zhengzhou University, Zhengzhou, China. ${ }^{2}$ Biological Psychiatry International Joint Laboratory of Henan/Zhengzhou University, Zhengzhou, China. ${ }^{3}$ Henan Psychiatric Transformation Research Key Laboratory/Zhengzhou University, Zhengzhou, China. ${ }^{4}$ Centre for Lifespan Changes in Brain and Cognition (LCBC), Department of Psychology, University of Oslo, Forskningsveien 3A, 0373 Oslo, Norway. ${ }^{5}$ Department of Psychiatry, The First Affiliated Hospital, Zhejiang University School of Medicine, Hangzhou, China. ${ }^{6}$ Chinese Research Academy of Environmental Sciences, Institute of Environmental Information, Beijing, China. ${ }^{7}$ Henan Gene Hospital, The First Affiliated Hospital of Zhengzhou University, Zhengzhou, China. ${ }^{8} \mathrm{Henan}$ Province Mental Hospital, The Second Affiliated Hospital/ Xinxiang Medical University, Xinxiang, China. ${ }^{9}$ Norwegian Centre for Mental Disorders Research (NORMENT), Institute of Clinical Medicine, University of Oslo, and Oslo University Hospital, Kirkeveien 166,0450 Oslo, Norway. ${ }^{10}$ Psychotic Disorders Program, UMass Memorial Medical Center/University of Massachusetts Medical School, Worcester, MA, USA. ${ }^{11}$ These authors contributed

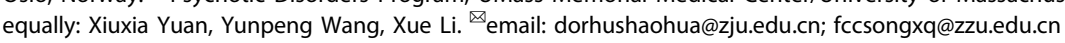

Received: 13 January 2021 Revised: 25 June 2021 Accepted: 15 July 2021

Published online: 10 August 2021 
Actinobacteria [9, 12, 18, 19] (e.g., Collinsella, Actinomyces, and Eggerthella) showed higher levels in $\mathrm{SCH}$ patients. Albeit inconsistent associations for specific microbial taxa, these studies indicated that patients with $\mathrm{SCH}$ displayed a dysbiosis of gut microbiota. Moreover, microbial biomarkers belonging to phylum Firmicutes, such as genera Lactobacillus, family Lachnospiraceae, Ruminococcaceae, Veillonellaceae, and Streptococcaceae were reported to be significantly correlated with symptom severity $[5,13,20]$. However, the role of gut microbiota in treatment response for $\mathrm{SCH}$ remains unclear.

Here, we present a 24-week study, including 107 first-episode, drug-naïve SCH patients, and 107 matched HCs. SCH patients were treated with risperidone monotherapy. Our primary goal was to identify the gut microbial biomarkers associated with the risperidone treatment response. We also would like to characterize microbiota in SCH patients. Inflammatory markers, hypersensitive C-reactive protein (hs-CRP), and homocysteine (HCY) (HCYinduced oxidative stress and inflammation via Nox4/NF-KB pathway) [21] have been reported to be associated with $\mathrm{SCH}[22,23]$, we additionally explored the relationship of gut microbiota, inflammatory markers, and treatment response.

\section{METHODS}

This study was approved by the Human Ethics Committee of the First Affiliated Hospital of the Zhengzhou University, China (Approval No. 2016LW-17). All enrolled first-episode, drug-naïve SCH patients have been carefully assessed by a psychiatrist between October 2017 and 2019. The following inclusion criteria were used: (1) diagnosis of $\mathrm{SCH}$ based on the Diagnostic and Statistical Manual of Mental Disorders fourth version (DSMIV) criteria, confirmed by the Structured Clinical Interview for DSM-IV (SCID) [24]; (2) medication free; (3) a Positive and Negative Syndrome Scale (PANSS) total score $>60$. Patients with the following conditions were excluded: (1) diagnosis of autoimmune diseases, heart diseases, hepatobiliary and gastrointestinal diseases, blood diseases, diabetes (type I and type II), neurological diseases, mental retardation, or other psychiatric diseases; (2) being pregnant or lactating; (3) treated with any antibiotics or anti-inflammatory agents in the previous month; (4) being obese (body mass index, $\mathrm{BMI}>28 \mathrm{~kg} / \mathrm{m}^{2}$ ) [25]. HCs were recruited from local communities through an online advertisement. $\mathrm{HCs}$ were matched to $\mathrm{SCH}$ patients by age, gender, education, smoking habits, and BMI. The same exclusion criteria applied to $\mathrm{SCH}$ patients were applied to HCs. In total, 107 patients and $107 \mathrm{HCs}$ at baseline were included (Supplementary Figure 1). All subjects provided signed informed consent.

\begin{abstract}
Assessments
After admission to the hospital, the clinical assessment was performed, and biological samples were collected before risperidone treatment. All subjects completed a comprehensive clinical interview, including the $\mathrm{SCID}$, and provided information on age, gender, education, smoking, disease duration, and medication history. Educations were calculated based on schooling years (including expected years of education, i.e., from the year of enrollment to the year of expected completion of education). Disease duration (days) was calculated as the time from the first appearance of the psychiatric symptoms to the first hospitalization. Before (week 0; S0), during (week 6 and 12; S1 and S2), and after (week 24; S3) treatment, study participants were assessed with PANSS, and side effects and somatic health screening. For SCH patients, the PANSS was performed at baseline, week 6, week 12, and week 24. The PANSS total scores (PANSST) include positive symptoms scores (PANSS-P), negative symptoms scores (PANSS-N), and general psychopathology symptoms scores (PANSS-G). PANSS factor scores for negative symptoms (PANSS-FSNS) and positive symptoms (PANSS-FSPS) were calculated as previously described [26]. The treatment response was defined as the mean change of PANSS-T scores from baseline to week 24 .
\end{abstract}

\section{Antipsychotic treatment}

Weighing the efficacy and side effects of antipsychotics [27] and clinical experience, risperidone (dopamine $D_{2}$ receptor and $5-\mathrm{HT}_{2}$ receptor antagonism) was used for pharmacological treatment. The dosage of risperidone was gradually titrated from $1 \mathrm{mg} /$ day to $4-6 \mathrm{mg} /$ day as clinically indicated. Clonazepam was used in three patients who had sleep problems. Eight patients had an extracorporeal vertebral response after risperidone was titrated to $6 \mathrm{mg} / \mathrm{d}$, and benzyl was used to improve the conditions. For various reasons, not all patients completed the full study. In the 6th-week, five patients did not provide stool samples; five patients did not come to our hospital due to unknown reasons, and one dropped due to hospitalization for poor treatment response. For the assessment at the 12th week, 10 patients did not come to our hospital due to unknown reasons, and 12 patients did not provide stool samples. In the 24th week, four patients did not come to our hospital, and four patients stopped medicine due to economic reasons. In total, $60 \mathrm{SCH}$ patients completed the 24-week study (Supplementary Figure 1).

\section{Biological samples collection}

Fresh fecal samples were collected at baseline, week 6, week 12, week 24 , and stored at $\mathrm{a}-80^{\circ} \mathrm{C}$ refrigerator immediately for the gut microbiota assay. The blood samples were collected at the same time for measuring inflammatory parameters, including hs-CRP and HCY. Serum levels of HCY were detected using an immune turbidimetric test (Roche Cobas C501, Switzerland). Serum levels of hs-CRP were measured by a chemical colorimetry assay and a particle-enhanced immunoturbidimetric assay respectively using a Roche automatic biochemical analyzer (Roche Diagnostics, C8000, Germany). All assays were performed according to the manufacturer's instructions. The gut microbiota and inflammatory biomarkers were measured with the subject's condition (patients vs. controls) blinded.

\section{DNA extraction}

DNA was extracted from $0.2 \mathrm{~g}$ of the fecal sample using the Cetyltrimethylammonium Ammonium Bromide CTAB/SDS method [28]. The purity and concentration of DNA were monitored using gel electrophoresis on $1 \%$ agarose gels. An amount of $150 \mu$ l of the DNA sample was obtained in the centrifuge tube and was diluted with sterile water to $1 \mathrm{ng} / \mu \mathrm{l}$.

\section{Amplicon generation}

The 16 S rRNA gene of distinct regions (V3-V4) was amplified using a specific primer with barcodes. The individually barcoded $341 \mathrm{~F}$ forward primers and the $806 \mathrm{R}$ reverse primers were CCTAYGGGRBGCASCAG and GGACTACNNGGGTATCTAAT, respectively. All PCRs were performed as $30 \mu \mathrm{L}$ reactions with $15 \mu \mathrm{L}$ of Phusion ${ }^{\circledast}$ High-Fidelity PCR Master Mix (New England Biolabs), and $0.2 \mu \mathrm{M}$ of forward and reverse primers and $10 \mathrm{ng}$ of template DNA. The reaction cycles consisted of $98^{\circ} \mathrm{C}$ predenaturation for $1 \mathrm{~min}$, followed by 30 cycles of $98^{\circ} \mathrm{C}$ denaturation for $10 \mathrm{~s}$, annealing at $50^{\circ} \mathrm{C}$ for $30 \mathrm{~s}$, elongation at $72{ }^{\circ} \mathrm{C}$ for $30 \mathrm{~s}$, and $72{ }^{\circ} \mathrm{C}$ elongation for $5 \mathrm{~min}$.

\section{PCR products purification}

After electrophoresis on $2 \%$ agarose gel, PCR products were purified using GeneJETTM Gel Extraction Kit (Thermo Scientific) and quantified according to the manufacturer's protocol and multiplexed at equal concentrations.

\section{Library preparation and sequencing}

The resultant library was sequenced on the lon S5 TM XL platform and $400 \mathrm{bp} / 600 \mathrm{bp}$ single-end reads were generated. Raw DNA sequences were quality controlled (QC) using Cutadapt (V1.9.1, http://cutadapt. readthedocs.io/en/stable/) [29]. Chimera sequences [30] were removed by comparing DNA sequences with the Silva reference database [31] using the UCHIME algorithm [32].

\section{Bioinformatic analysis}

Sequence analyses were performed using the Uparse software (V7.0.1001) [33]. A widely employed similarity threshold of $97 \%$ was used to assign quality-controlled sequences to operational taxonomic units (OTUs). The taxonomy of microbes was determined using the Mothur algorithm (https://www.mothur.org) based on the Silva Database (https://www.arbsilva.de/) [31].

a-diversity was estimated using the Shannon index, Simpson index, Polygenetic Diversity Index (PD whole tree), Observed Species index, Chao index, and Abundance Coverage-based Estimator (ACE index) using QIIME (V1.7.0). $\beta$-diversity was computed based on the Bray Curtis Dissimilarity, Binary Jaccard Dissimilarity, Weighted and Unweighted UniFrac Distance. the former two calculation does not consider phylogenetic relatedness of taxa but the latter two calculation does. The difference in $\beta$-diversity 
between SCH patients and HCs was examined using permutational multivariate analysis of variance (PERMANOVA). Principal Coordinate Analysis (PCOA) was performed to obtain principal coordinates. PERMANOVA was conducted using the vegan package of the $R$ software (V3.6.2). PCoA results were displayed using the WGCNA package, the stat packages, and the ggplot2 package of the R software (V3.6.2).

Microbial Dysbiosis Index (MD index) was determined using log $(A / B)$, where $A$ is the total abundance of genera that was higher in $\mathrm{SCH}$ patients than in HCs; B is the total abundance of genera that was lower in $\mathrm{SCH}$ patients than in HCs [34].

\section{Statistical analyses}

For continuous variables, within each group, a normality test was performed using the Shapiro-Wilk test, the homogeneity of variance was performed using Levene's test. For categorical variables, the Chi-squared test was used to check for independence among variables. In all statistical analyses, age, gender, education, disease duration, smoking status, and BMI were used as covariates. False discovery rate (FDR) [35] was used to correct multiple testing in the present study.

\section{Differences in microbial biomarkers between $\mathrm{SCH}$ patients and HCs at baseline}

The Wilcoxon test was used to identify differential abundant bacteria between patients and HCs. Microbial biomarkers that have a mean abundance $\geq 0.001$ and $\geq 60 \%$ of samples with the abundance of microbial biomarkers $\geq 0.001$, were analyzed in the present study.

The Random Forest methods [36] (randomForest package [37] from $R$ V3.6.2) were used to construct prediction models for SCH using differential abundant microbial biomarkers between patients and $\mathrm{HCs}$. A cross-validation procedure was employed to evaluate the generalization performance of the resultant prediction model. Two-thirds of the samples were randomly selected as training set to build the prediction models; the remaining $1 / 3$ samples were used as the verification set. This procedure was repeated 1000 times, and the average values for the performance metrics were used as indicators for the predictive performance of the model. Gini importance (or mean decrease impurity) is computed from the Random Forest method to describe which features are relevant. The receiver operating characteristic (ROC) curve function from the randomForest package was used to compute the area under the curve (AUC).

\section{Changes in gut microbiota and clinical symptoms after risperidone treatments in SCH patients}

The linear discriminant analysis (LDA) effect-size (LEfSe) method was used to perform metagenomics analysis [38, 39]. LDA was used to reduce the dimensionality of the data and evaluate the influence of the statistically different microbes (LDA score). The default parameters of the LEfSe package were used, alpha $=0.05$ and effect-size threshold $=3.0$. The paired Wilcoxon test was used to test the changes in the differential abundant bacteria, which were identified at baseline in $\mathrm{SCH}$ patients, at different time points after risperidone treatment. A similar procedure was applied for the PANSS scores and inflammatory biomarker hs-CRP and HCY measured in this study.

\section{Correlations between microbial biomarkers and the PANSS total scores}

The partial correlations between the severity of symptoms and microbial biomarkers that were different between $\mathrm{SCH}$ and $\mathrm{HCS}$ at baseline and/or significantly changed after risperidone treatment in patients were also analyzed after controlling for various covariates.

\section{Relationship between the changes in the PANSS total scores and the changes in microbial biomarkers}

The relationship between the changes of the PANSS total scores and the changes in microbial biomarkers that were different between $\mathrm{SCH}$ and $\mathrm{HCs}$ at baseline and/or significantly changed after risperidone treatment were examined using stepwise linear regression models using the $F$ test with parameters, $p$ value threshold in $=0.05$ and threshold out $=0.10$. The changes in the PANSS total scores were set as dependent variables, the changes in microbial biomarkers and inflammatory biomarkers (hs-CRP and $\mathrm{HCY}$ ), and various covariates were set as independent variables.
Baseline microbial biomarker levels and treatment response in SCH patient

The relationship between the treatment response and the gut microbial biomarkers that were different between $\mathrm{SCH}$ and $\mathrm{HCs}$ at baseline and/or significantly changed at different time points in patients were explored using random-intercept linear mixed-effect models. The changes of the PANSS total scores at each time point were set as dependent variables and the time point, baseline microbial biomarker levels, and various covariates were set as fixed-effect predictors. The participant identifiers were used as a random variable in the models. The Ime4 [40] and the ImeTest packages from R (V3.6.2) were used for these analyses. For mixed-effect models, the approximated $p$ values $<0.05$ were considered statistically significant.

\section{RESULTS}

\section{Clinical characteristics and evaluation}

There were no significant differences in age, gender, education, smoking, and BMI between the SCH patients and $\mathrm{HCs}$ (all $p>0.05$ ) (Table 1). We observed higher levels of hs-CRP and $\mathrm{HCY}$ in the $\mathrm{SCH}$ group than in HCs at baseline ( $p=0.035,1.49 \times 10^{-6}$, respectively) (Table 1).

After 24 weeks of risperidone treatment, serum HCY levels decreased and hs-CRP and BMI increased significantly (all $p<0.05$, Fig. 1). In addition, clinical improvement was observed as reflected by the reduction in the PANSS scores (all $p<0.05$, Fig. 1).

After 24 weeks of treatment, the baseline levels of hs-CRP were positively correlated with the changes of the PANSS-T scores ( $p=$ 0.015). The baseline levels of HCY were also positively correlated with the changes of the PANSS-FSPS $(p=0.012)$.

\section{Microbial biomarker annotation}

In total, 472 samples from all recruited participants were sequenced using lonS5 ${ }^{\mathrm{TM}} \mathrm{XL}$ sequencer platform. From 472 samples, 76,747 high-quality reads were obtained. These reads were clustered into 8450 qualified OTUs at $97 \%$ sequence similarity and $8433(99.80 \%)$ of them could be annotated to the database. 95.17 84.53 , and $61.51 \%$ of all reads were annotated to phylum, family, and genus. In order to ensure the accuracy and reliability of sequencing data, 28 duplicated samples were randomly selected and sequenced. Paired sample $T$-test showed no significant differences in the diversity and composition of intestinal microorganisms between the two groups of samples (Supplementary Table 1). Finally, 444 samples were further analyzed.

A Venn diagram showed that 2223 of 5416 OTUs were detected in the two groups, while 2270 and 923 OTUs were unique to patients with $\mathrm{SCH}$ and $\mathrm{HCs}$, respectively (Supplementary Figure $2 \mathrm{~A})$. Most rarefaction curves tended to approach the saturation plateau, suggesting that the sequencing depth was enough to cover the whole bacterial diversity (Supplementary Figure 2B). The box plot tended to approach the saturation plateau, indicating that the sample capacity was sufficient for data analysis (Supplementary Figure 2C). The predominant phylum and genus bacteria in $\mathrm{SCH}$ and $\mathrm{HCs}$ group were shown in Supplementary Figure 2D, E.

\section{Change in microbial a-diversity in SCH patients}

We analyzed six a-diversity indices of $\mathrm{SCH}$ patients and $\mathrm{HCs}$ based on the OTU relative table. We analyzed Shannon index and Simpson index, which reflect richness and evenness. We found significant lover levels of Shannon and Simpson's indices in $\mathrm{SCH}$ patients than in $\mathrm{HCs}\left(p=1.21 \times 10^{-9}, 1.23 \times 10^{-8}\right.$, respectively) (Fig. 2A, B). PD whole tree index incorporates phylogenetic differences, and the phylogenetic diversity reflects the phylogenetic distance of each species in a community. The more complex the species' kinship within the community is, the farther the evolutionary distance and the higher levels of phylogenetic diversity are. When using phylogenetic diversity, we observed a significant increase in this measure in the $\mathrm{SCH}$ patients than in $\mathrm{HCs}$ (Fig. 2C). Both observed species index and Chao1 index reflect 
Table 1. Demographic characteristics and statistical analysis.

\begin{tabular}{|c|c|c|c|c|}
\hline & $\begin{array}{l}\text { SCH patients }(N=107) \\
\text { Median (IOR) }\end{array}$ & $\begin{array}{l}\text { Healthy controls }(N=107) \\
\text { Median }(\text { IOR) }\end{array}$ & $Z, t$, or $X^{2}$ value & $p$ value \\
\hline Age (years) & $19(19-25)$ & $23.00(22.00-25.00)$ & 1.642 & 0.103 \\
\hline Education years & $12(11-13)$ & $12(12-12)$ & -0.654 & 0.513 \\
\hline Body weight (kg) & $58.6(51-65)$ & $57.15(52.3-67.5)$ & -0.579 & 0.563 \\
\hline BMI $\left(\mathrm{kg} / \mathrm{m}^{2}\right)$ & $20.71(18.91-22.41)$ & $21.17(19.41-23.16)$ & -1.373 & 0.170 \\
\hline hs-CRP (mg/L) & $0.28(0.15-0.62)$ & $0.20(0.13-0.40)$ & -1.995 & 0.035 \\
\hline PANSS-FSNS & $19.00(14.75-23.00)$ & & & \\
\hline PANSS-G & $39.00(35.00-47.00)$ & & & \\
\hline PANSS-T & $79.50(72.00-89.25)$ & & & \\
\hline Disease duration & $180(30-365)$ & & & \\
\hline \multicolumn{5}{|l|}{ Gender } \\
\hline Yes & $5(5 \%)$ & $6(6 \%)$ & 0.096 & 0.500 \\
\hline No & 102 (95\%) & 101 (94\%) & & \\
\hline
\end{tabular}

Note: Hs-CRP high-sensitivity C-reactive protein, HCY homocysteine, PANSS-FSPS PANSS factor score for positive symptoms, PANSS-FSNS PANSS factor score for negative symptoms, PANSS The Positive and Negative Syndrome Scale, including positive, negative, and general psychopathology symptoms represented by PANSS-P, PANSS-N, and PANSS-G, respectively, PANSS-T=PANSS-P+PANSS-N+PANSS-G, SCH schizophrenia.

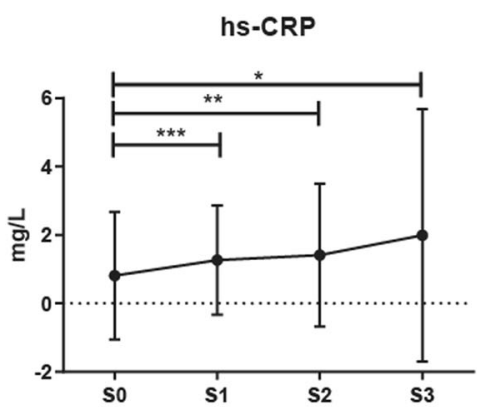

PANSS-FSPS

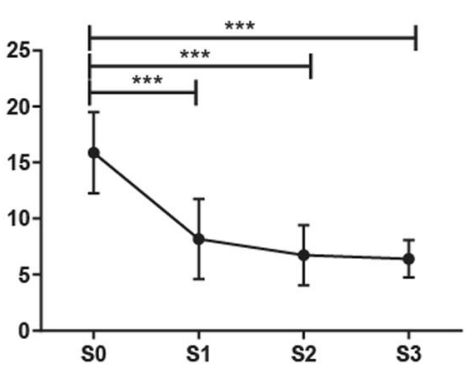

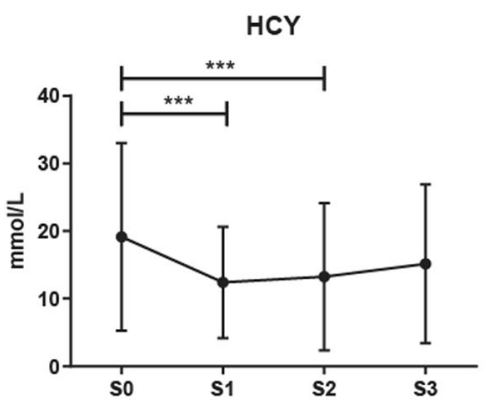
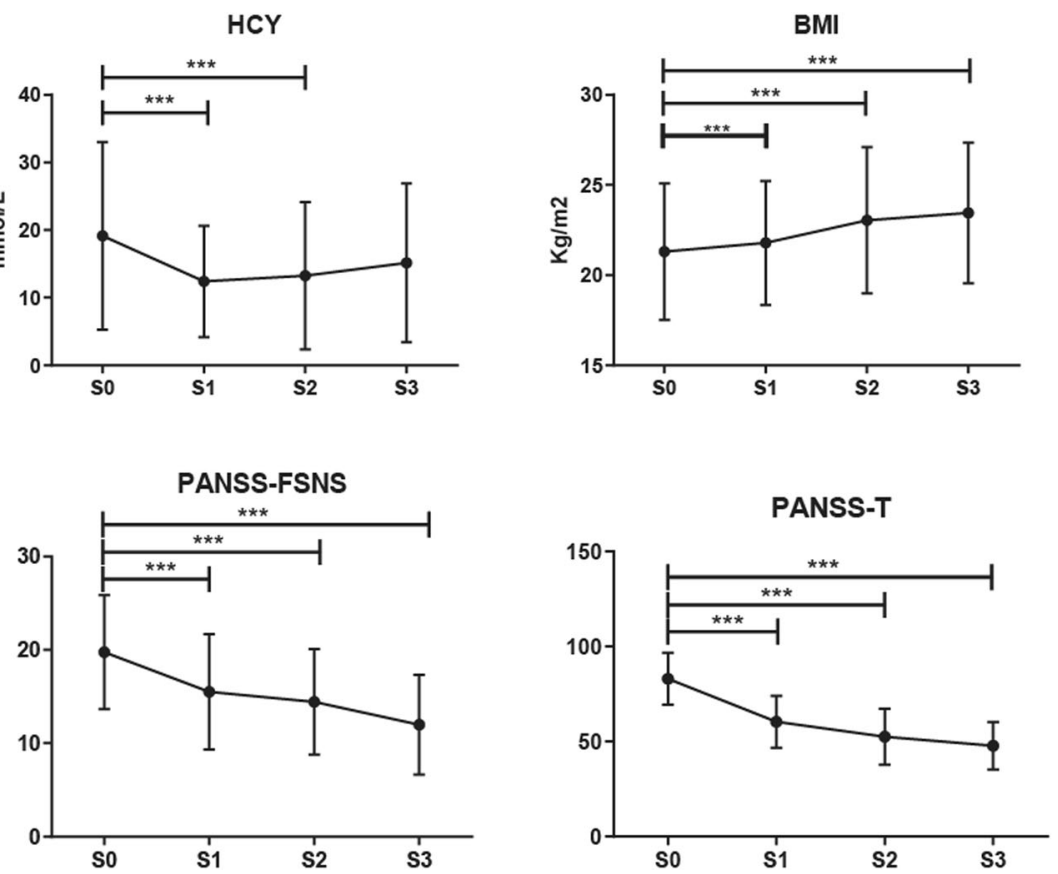

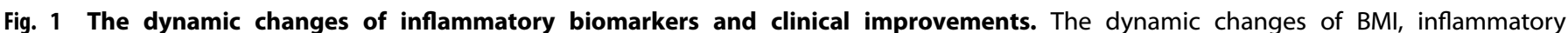

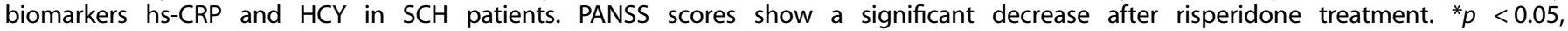
${ }^{* *} p<0.01,{ }^{* *} p<0.001$. The means and standard deviation are indicated in the bar chart. FDR were used to correct the multiple testing.

species richness; the Chao1 index can reflect the existence of low abundance species in the community. Abundance Coveragebased Estimator (ACE) reflects the total number of species, considering both species abundance and the probability of species in the sample. In these measures, there were no significant differences between SCH patients and HCs (Fig. 2D-F). Our result suggests that the internal similarity of the bacterial community in $\mathrm{SCH}$ patients is higher than in $\mathrm{HCs}$.

Change in microbial $\boldsymbol{\beta}$-diversity in $\mathbf{S C H}$ patients

We then analyzed the $\beta$-diversity to evaluate whether the whole microbiome community was different between $\mathrm{SCH}$ patients and 


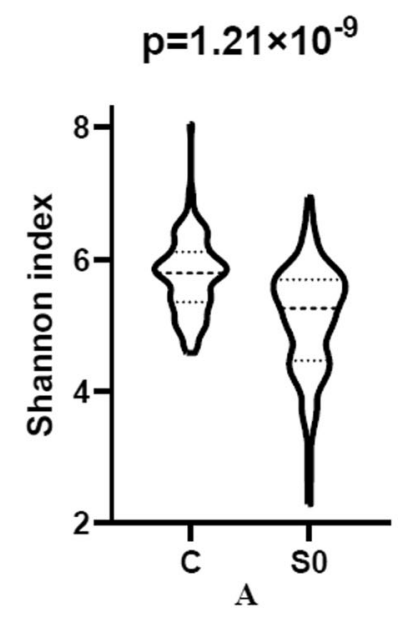

$\mathrm{p}=0.767$

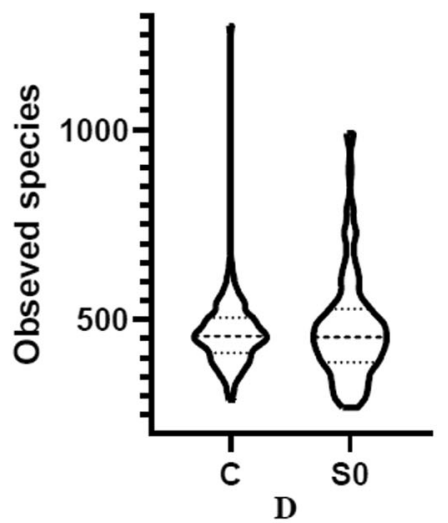

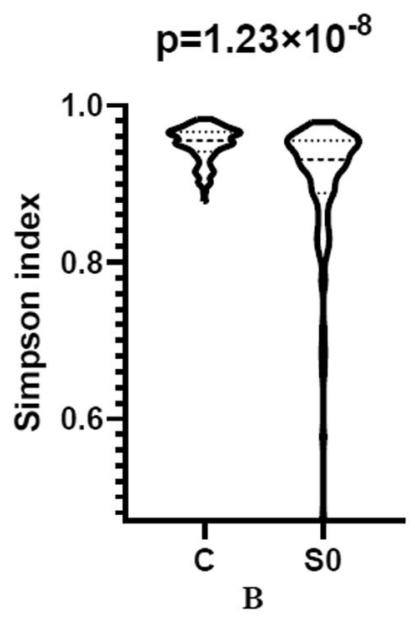

$p=0.452$

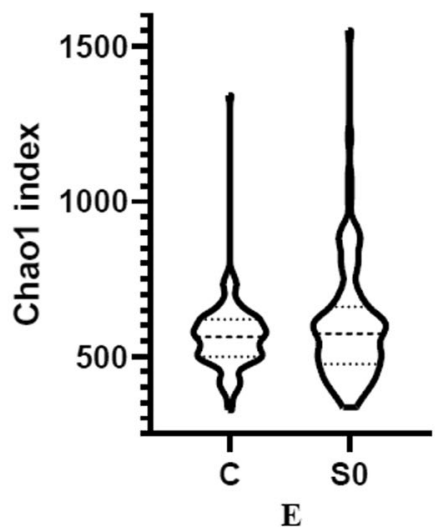

$p=0.005$

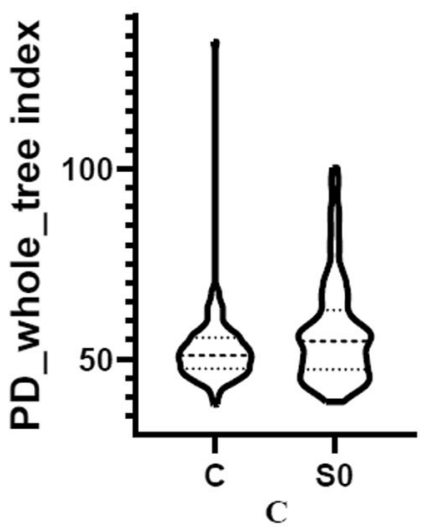

$\mathrm{p}=\mathbf{0 . 2 5 5}$

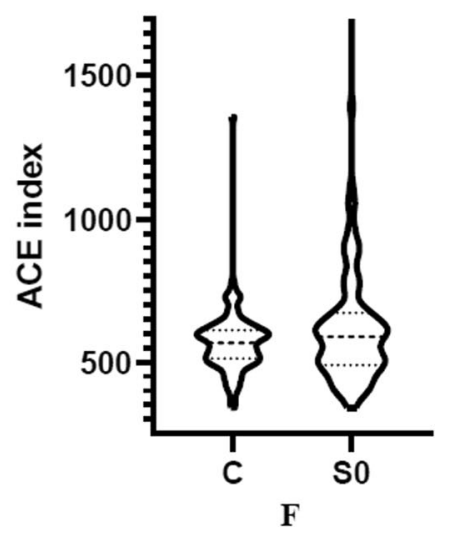

Fig. 2 The alteration of a diversity in SCH patients. S0: schizophrenia, C: healthy controls. FDR were used to correct the multiple testing.

HCs. We found a significant difference in bacterial community composition between SCH patients and HCs based on BrayCurtis and Binary Jaccard dissimilarity calculations, which do not take phylogenetic relatedness of taxa into consideration (Supplementary Figure 3A, B). Similarly, there was a significant difference in the composition of bacterial gut microbiota based on weighted and unweighted UniFrac distances, which take phylogenetic relatedness into account (Supplementary Figure 3C, D). The PERMANOVA based on those four $\beta$-diversity dissimilarity metrics shows that the between-group difference was significantly different than the within-group difference, in either $\mathrm{SCH}$ patients or $\mathrm{HCs}$ (all $R^{2}>0, p<0.05$ ) (Supplementary Table 2); accordingly, Principal Coordinate Analysis (PCoA) based on the four metrics showed a significant separation of the two groups (Supplementary Figure 4A-D). These results indicating a significant difference in bacterial community composition between $\mathrm{SCH}$ patients and $\mathrm{HCs}$.

\section{Dysbiosis of gut microbiota in SCH patients}

We discovered 19 genera that were significantly different between $\mathrm{SCH}$ patients and $\mathrm{HCs}$ (all $\mathrm{p}<0.05$ ). The genus Lachnoclostridium showed higher levels and the other 18 genera showed lower levels in $\mathrm{SCH}$ patients than in $\mathrm{HCs}$ (all $p<0.05$ ) (Supplementary Table 3). Of note, 14 of these genera (73.7\%) are members of the phylum Firmicutes. We observed a significantly higher level of MD index in SCH patients than in $\mathrm{HCs}(p=1.39 \times$ $10^{-15}$ ) (Fig. 3A). We constructed a Random Forest predictive model for $\mathrm{SCH}$ patients using the abundance of these 19 microbial biomarkers as predictors. In the discovery set (on average, $82 \mathrm{SCH}$ patients and $86 \mathrm{HCs}$ ), a predictive model of 10 microbial biomarkers (Dorea, Romboutsia, Streptococcus, Blautia, Anaerostipes, Terrisporobacter, Weissella, Alistipes, Haemophilus, and Lachnoclostridium) showed a remarkable discriminating power, with an AUC of 0.879 (Fig. 3B). In the verification set (on average, $25 \mathrm{SCH}$ patients and $21 \mathrm{HCs}$ ), the model effectively differentiated $\mathrm{SCH}$ patients from $\mathrm{HCs}$ with an AUC of 0.867 (Fig. 3C). These 10 microbial biomarkers are shown in Fig. 3D in descending order of Gini importance.

\section{Changes of gut microbiota in SCH patients}

We observed significant changes in microbial diversity and microbial abundance during the 24 weeks of risperidone treatment. The a-diversity (both Shannon and Simpson's) indices of SCH patients increased ( $p=0.046$ and 0.057 , respectively) after 24 weeks of treatment, but were still lower than the baseline levels of HCs (all $p<0.05$ ) (Fig. 4A, B). To identify the effect of risperidone treatment on whole microbiota composition in $\mathrm{SCH}$ patients, we used the LEfSe algorithm to compare microbial biomarkers before and after risperidone treatment (Supplementary Figure 5A, B). A total of 14 taxonomies had significantly different levels across different time points. Lachnoclostridium was enriched in patients before risperidone treatment. After 24 weeks of treatment, the gut microbiota in patients was dominated by Lachnospira, Sutterella, and Alistipes. Among the 19 microbial biomarkers discovered at baseline, two genera showed changes compared to the baseline levels. Specifically, Lachnoclostridium decreased $(p=0.019)$ and 

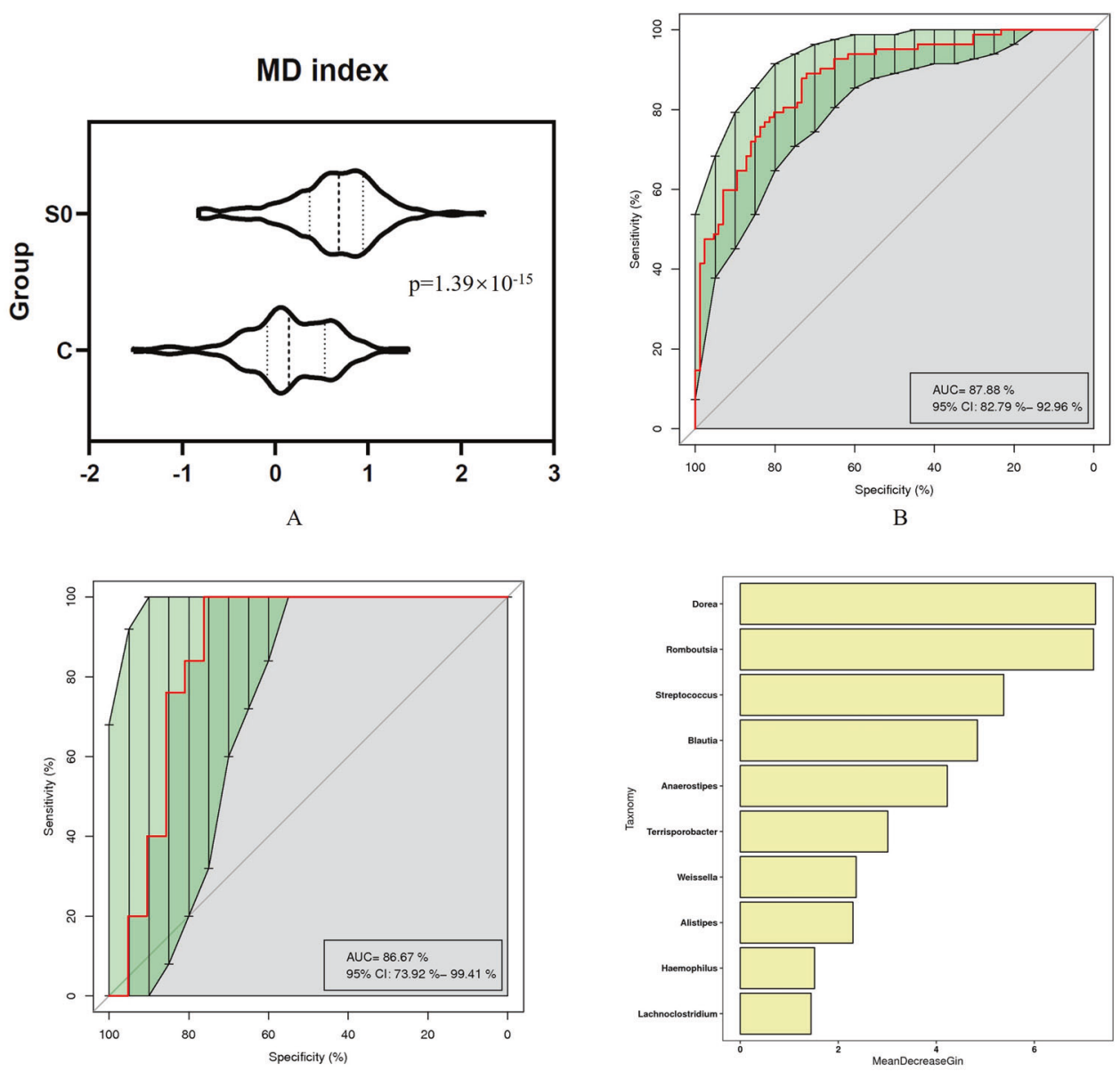

$\mathrm{C}$

$\mathrm{D}$

Fig. 3 Alteration of microbial biomarkers in SCH patients. A Comparison of MD index between SCH patients and HCs. B and C prediction models for discovery samples B and testing samples C. D The 10 microbial biomarkers in descending order of Gini coefficient. S0: SCH patients, C: healthy controls. FDR were used to correct the multiple testing.

Romboutsia increased after risperidone treatment $(p=0.067)$ (Fig. 4C, D). At week 24, 14 out of these 19 biomarkers show significantly lower abundance levels in $\mathrm{SCH}$ patients compared with HCs (all $p<0.05$ ).

\section{Relationship between microbial biomarkers and clinical symptoms of SCH at baseline}

The two genera, Lachnoclostridium and Romboutsia, and phylum Firmicutes [11, 41] were tested for their association with the severity of $\mathrm{SCH}$ symptoms at baseline. After controlling for various covariates in partial correlation analysis, the Lachnoclostridium abundance was positively correlated with the PANSS-FSNS $(r=$ $0.458, p=0.002$ ); the abundance of Firmicutes was found to be positively correlated with the PANSS-FSNS $(r=0.384, p=0.014)$. These associations remained to be significant after inflammatory biomarkers and MD index were included as additional covariates.

\section{Relationship between microbial biomarkers and treatment response}

The two genera, Lachnoclostridium and Romboutsia, were also examined for their associations with the treatment response at week 24. After controlling for various covariates, the changes in Lachnoclostridium were positively correlated with the changes in the PANSS-FSNS ( $p=0.006$ ) (Fig. 4E). The changes in Romboutsia $(p=0.002)$ were negatively correlated with the changes in PANSSFSNS (Fig. 4F). In addition, we found that higher baseline levels of Romboutsia and Lachnoclostridium were associated with poor treatment response ( $p=0.006$ and 0.005 , respectively).

\section{DISCUSSION}

In the present study, we found a significant relationship between microbial biomarkers and clinical response after 24-week risperidone treatment in the first-episode, drug-naïve $\mathrm{SCH}$ patients. These findings suggest a role of the gut microbiota in $\mathrm{SCH}$ psychopathology and indicate that microbiota measures may serve as biomarkers in $\mathrm{SCH}$ treatment.

Our results are consistent with previous findings [5, 9]. Bacterial diversity in the gut measured by Shannon and Simpson's indices (richness and evenness) is generally thought to be related to health [42], and low a-diversity has been associated with a range of chronic human diseases $[43,44]$. Several studies have shown a lower microbial a-diversity in $\mathrm{SCH}$ patients than in $\mathrm{HCs}[5,9]$. Here, we further suggested that low a-diversity is associated with disease severity of $\mathrm{SCH}$, such as positive symptoms. $\beta$-diversity presents the similarity or dissimilarity between two or more sets of microbial communities. Consistent with previous reports $[5,12,20]$, our study shows that at the community level, SCH patients may have a distinct intestinal ecological state than in HCs $[5,12,20]$. Our findings were also in line with previous reports at several taxonomic levels in SCH patients [45]. However, some other studies found no significant difference in a-diversity between SCH patients and HCs $[12,20,45]$. The discrepant findings may be attributed to the differences in participants (first episode vs. chronic disease phase, antipsychotic medication exposure, geographic locations, and lifestyle).

We discovered 19 genera that showed significant differences between $\mathrm{SCH}$ patients and $\mathrm{HCs}$ at baseline. Five of them were 

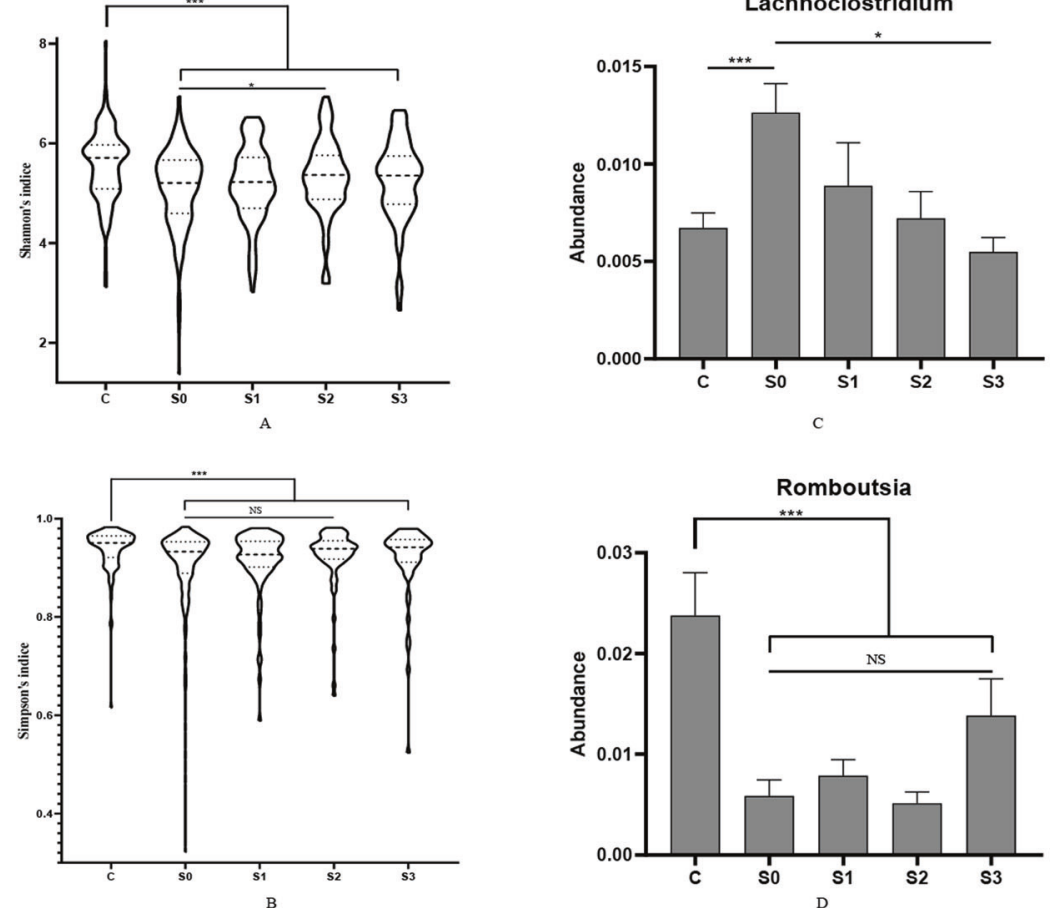
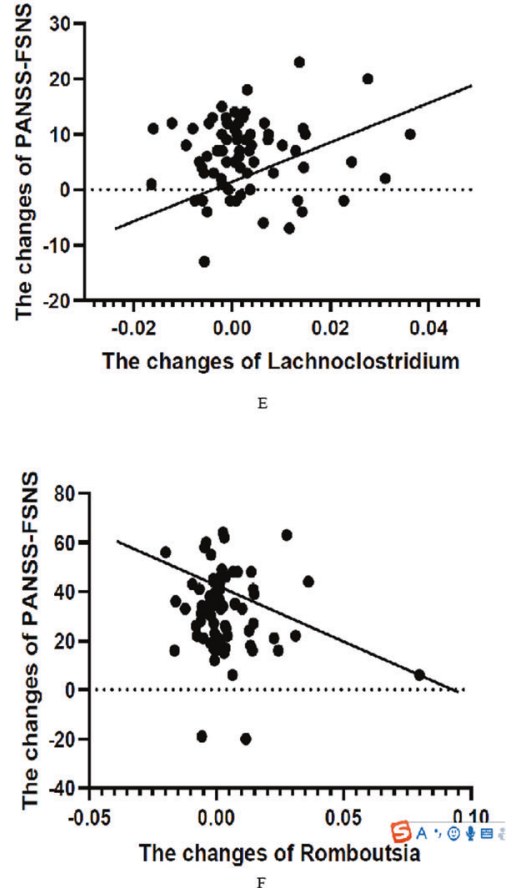

Fig. 4 Dynamic changes of gut microbiota in SCH patients. A and $\mathbf{B} \alpha$ diversity, is lower in SCH patients than in HCs (indicated by C) at baseline and was increased after risperidone treatment in SCH patients. C and D SCH patients show a higher level of Lachnoclostridium and a lower level of Romboutsia than HCs. E and F The correlation between the changes of Lachnoclostridium, Romboutsia and clinical improvement measured by PANSS-FSNS. FDR were used to correct the multiple testing. ${ }^{*} p<0.05,{ }^{* *} p<0.01,{ }^{* * *} p<0.001$.

consistent with the findings from previous studies, i.e., Streptococcus, Haemophilus, Enterococcus, Blautia, and Bifidobacterium $[9,11,15,20,23]$. In addition, the abundance of the Lachnospiraceae family was reported to be decreased in $\mathrm{SCH}$ [5]. Our study found that six genera (Blautia, unidentified_Lachnospiraceae, Anaerostipes, Dorea, Fusicatenibacter, and Butyrivibrio) that belong to family Lachnospiraceae decreased in $\mathrm{SCH}$ patients. The remaining nine biomarkers discovered in our study have not been reported previously. Most previous studies have reported the impact of disease duration and antipsychotic medication uses on the composition of gut microbiota [46, 47], our study minimized such confounding effects. We found that microbiota from Firmicutes may play an important role in the pathogenesis of $\mathrm{SCH}$ and other psychiatric diseases [48-50]. Studies from our group have demonstrated a significant role of immune and inflammatory processes, such as increased levels of hs-CRP and $\mathrm{HCY}$, in the risk of $\mathrm{SCH}[51,52]$. Both gut microbiota and inflammation have been associated with the pathogenesis of $\mathrm{SCH}$ [9]. The dysbiosis of gut microbiota could lead to inflammation in several diseases [53]. We did not find significant correlations between hs-CRP, HCY, and any of the 19 differential genera. This may be attributed to the complex relationships between inflammation and gut microbiota in $\mathrm{SCH}$. Immune dysfunction associated with the changes in the gut ecosystem may not necessarily reflected by the levels of hs-CRP and HCY may by perturbed by changes in gut ecosystems. Future studies are warranted to answer questions, such as whether abnormal gut microbiota causes a dysfunction of the immune response, or whether dysfunction of the immune response affects the gut ecosystems, therefore resulting in an increased risk of $\mathrm{SCH}$.

Our study also proposed a prediction model of 10 microbial biomarkers, which showed high accuracy (AUC $=0.879$ ), and validated in the verification set (AUC $=0.867$ ), indicating a powerful classification model. Previous studies have shown that SCH patients contain harmful microbial species that may contribute to $\mathrm{SCH}$ symptoms. Five microbial biomarkers (Dorea, Streptococcus, Blautia,
Anaerostipes, and Haemophilus) in the present study were reported in previous studies $[12,14,19,20,20]$. Future studies should further confirm the clinical utility of our predictive model.

Our results suggest an increased a-diversity in $\mathrm{SCH}$ patients after risperidone treatment. A recent study in an ex vivo fermentation assay showed that, after $24 \mathrm{~h}$ of incubation, risperidone was completely decomposed to ketone, suggesting that microbiota-based biotransformation of risperidone [54]. Notably, we showed that the baseline levels of Lachnoclostridium, Romboutsia, both belonging to Firmicutes, could be used to predict the treatment response. The genus Lachnoclostridium was elevated in $\mathrm{SCH}$ at baseline and reversed after risperidone treatment. The changes of this genera were positively correlated with the changes in clinical symptoms.

Previous studies have reported the effect of gut microbes on the function of the neurotransmitter systems and amino acid metabolism [5, 10]. The level of Firmicutes could impact the function of the dopamine pathway through its modulation on the level of dopamine $\beta$-hydrolase [55]. Moreover, low levels of Romboutsia, a short-chain fatty acid (SCFA) producing bacteria, were associated with the disturbances of glutamate/GABAglutamine cycle and astrocyte-neuron metabolism system [55]. In addition, Lachnoclostridium has been reported to affect the tryptophan metabolism [56]. Therefore, microbial biomarkers for $\mathrm{SCH}$ discovered in our study can affect the function of the neurotransmitter systems and amino acid metabolism as reported in previous studies [57].

The present study has several strengths. $\mathrm{SCH}$ patients and $\mathrm{HC}$ subjects were demographically matched by age, gender, education, smoking habits, and BMI. Only the first-episode and drugnaïve $\mathrm{SCH}$ patients were included in this study. Thus, confounding effects from antipsychotic treatment and disease durations were largely eliminated. We confirmed the stability of our sampling and $16 \mathrm{~s}$ RNA gene sequencing protocols by analyzing 28 duplicated samples. In addition, the gut microbiota was tested with the participant status (SCH patients vs $\mathrm{HCs}$ ) blinded. Furthermore, our 
study design followed the patients for a relatively longer period than previous studies [45]. However, our study also has limitations. We did not follow the matched HCs. The stochastic fluctuations in the changes of microbial marker levels in SCH patients could not be controlled. However, our relatively larger sample size may overweigh these random noises inherent in the data. We only focused on risperidone treatment of $\mathrm{SCH}$. Therefore, our results may not be generalizable to treatment with other antipsychotic medications.

In conclusion, we characterized potential microbial biomarkers in the first-episode, drug-naïve $\mathrm{SCH}$ patients and their role in relation to clinical response to risperidone treatment Our results suggest a role of microbiota in $\mathrm{SCH}$ pathobiology. Novel intervention strategies that target microbiota might be promising in schizophrenia treatment.

\section{REFERENCES}

1. Lee J, Green MF. Social preference and glutamatergic dysfunction: underappreciated prerequisites for social dysfunction in schizophrenia. Trends Neurosci. 2016;39:587-96.

2. Falkai P, Rossner MJ, Schulze TG, Hasan A, Brzózka MM, Malchow B, et al. Kraepelin revisited: schizophrenia from degeneration to failed regeneration. Mol Psychiatry. 2015;20:671-76.

3. Nimgaonkar VL, Prasad KM, Chowdari KV, Severance EG, Yolken RH. The complement system: a gateway to gene-environment interactions in schizophrenia pathogenesis. Mol Psychiatry. 2017;22:1554-61.

4. Stan AD, Ghose S, Zhao C, Hulsey K, Mihalakos P, Yanagi M, et al. Magnetic resonance spectroscopy and tissue protein concentrations together suggest lower glutamate signaling in dentate gyrus in schizophrenia. Mol Psychiatry. 2015;20:433-39.

5. Zheng $P$, Zeng B, Liu M, Chen J, Pan J, Han Y, et al. The gut microbiome from patients with schizophrenia modulates the glutamate-glutamine-GABA cycle and schizophrenia-relevant behaviors in mice. Sci Adv. 2019;5:eaau8317.

6. Kindler J, Lim CK, Weickert CS, Boerrigter D, Galletly C, Liu D, et al. Dysregulation of kynurenine metabolism is related to proinflammatory cytokines, attention, and prefrontal cortex volume in schizophrenia. Mol Psychiatry. 2020;25:2860-72.

7. Cervenka I, Agudelo LZ, Ruas JL. Kynurenines: tryptophan's metabolites in exercise, inflammation, and mental health. Science. 2017;357:eaaf9794.

8. Israelyan N, Del Colle A, Li Z, Park Y, Xing A, Jacobsen J, et al. Effects of serotonin and slow-release 5-hydroxytryptophan on gastrointestinal motility in a mouse model of depression. Gastroenterology. 2019;157:507-21.e504.

9. Xu R, Wu B, Liang J, He F, Gu W, Li K, et al. Altered gut microbiota and mucosal immunity in patients with schizophrenia. Brain Behav Immun. 2020;85:120-27.

10. Zhu F, Ju Y, Wang W, Wang Q, Guo R, Ma Q, et al. Metagenome-wide association of gut microbiome features for schizophrenia. Nat Commun. 2020;11:1612.

11. Castro-Nallar E, Bendall ML, Pérez-Losada M, Sabuncyan S, Severance EG, Dickerson $\mathrm{FB}$, et al. Composition, taxonomy and functional diversity of the oropharynx microbiome in individuals with schizophrenia and controls. PeerJ. 2015;3: e1140.

12. Shen Y, Xu J, Li Z, Huang Y, Yuan Y, Wang J, et al. Analysis of gut microbiota diversity and auxiliary diagnosis as a biomarker in patients with schizophrenia: a cross-sectional study. Schizophr. Res. 2018;197:470-77.

13. Schwarz E, Maukonen J, Hyytiäinen T, Kieseppä T, Orešič M, Sabunciyan S, et al. Analysis of microbiota in first episode psychosis identifies preliminary associations with symptom severity and treatment response. Schizophr. Res. 2018;192:398-403.

14. Li S, Zhuo M, Huang X, Huang Y, Zhou J, Xiong D, et al. Altered gut microbiota associated with symptom severity in schizophrenia. PeerJ. 2020;8:e9574.

15. Huang Y, Shi X, Li Z, Shen Y, Shi X, Wang L, et al. Possible association of Firmicutes in the gut microbiota of patients with major depressive disorder. Neuropsychiatr Dis Treat. 2018;14:3329-37.

16. Lin P, Ding B, Feng C, Yin S, Zhang T, Qi X, et al. Prevotella and Klebsiella proportions in fecal microbial communities are potential characteristic parameters for patients with major depressive disorder. J Affect Disord. 2017;207:300-04.

17. Iglesias-Vázquez L, Van Ginkel Riba G, Arija V, Canals J. Composition of gut microbiota in children with autism spectrum disorder: a review and metaanalysis. Nutrients. 2020;12:792.

18. Pan R, Zhang X, Gao J, Yi W, Wei Q, Su H. Analysis of the diversity of intestinal microbiome and its potential value as a biomarker in patients with schizophrenia: a cohort study. Psychiatry Res. 2020;291:113260.
19. Zhang X, Pan LY, Zhang Z, Zhou YY, Jiang HY, Ruan B. Analysis of gut mycobiota in first-episode, drug-naïve Chinese patients with schizophrenia: a pilot study. Behavioural Brain Res. 2020;379:112374.

20. Nguyen TT, Kosciolek T, Maldonado Y, Daly RE, Martin AS, McDonald D, et al. Differences in gut microbiome composition between persons with chronic schizophrenia and healthy comparison subjects. Schizophrenia Res. 2019;204:23-9.

21. Hu H, Wang C, Jin Y, Meng Q, Liu Q, Liu Z, et al. Catalpol inhibits homocysteineinduced oxidation and inflammation via inhibiting Nox4/NF-KB and GRP78/PERK pathways in human aorta endothelial cells. Inflammation. 2019;42:64-80.

22. Fond G, Lançon C, Auquier P, Boyer L. C-reactive protein as a peripheral biomarker in schizophrenia. An updated systematic review. Front Psychiatry. 2018;9:392.

23. Yuan X, Zhang P, Wang Y, Liu Y, Li X, Kumar BU, et al. Changes in metabolism and microbiota after 24-week risperidone treatment in drug-naïve, normal weight patients with first episode schizophrenia. Schizophrenia Res. 2018;201:299-306.

24. Runeson BS, Rich CL. Diagnostic and statistical manual of mental disorders, 3rd ed. (DSM-III), adaptive functioning in young Swedish suicides. Ann Clin Psychiatry. 1994;6:181-3.

25. Yang Z, Ding X, Liu J, Duan P, Si L, Wan B, et al. Associations between anthropometric parameters and lipid profiles in Chinese individuals with age $\geq 40$ years and $\mathrm{BMI}<28 \mathrm{~kg} / \mathrm{m}^{2}$. PLOS ONE. 2017;12:e0178343.

26. Németh G, Laszlovszky I, Czobor P, Szalai E, Szatmári B, Harsányi J, et al. Cariprazine versus risperidone monotherapy for treatment of predominant negative symptoms in patients with schizophrenia: a randomised, double-blind, controlled trial. Lancet. 2017;389:1103-13.

27. Huhn M, Nikolakopoulou A, Schneider-Thoma J, Krause M, Samara M, Peter N, et al. Comparative efficacy and tolerability of 32 oral antipsychotics for the acute treatment of adults with multi-episode schizophrenia: a systematic review and network meta-analysis. Lancet. 2019;394:939-51.

28. Griffith GW, Ozkose E, Theodorou MK, Davies DR. Diversity of anaerobic fungal populations in cattle revealed by selective enrichment culture using different carbon sources. Fungal Ecol. 2009;2:87-97.

29. Martin M. Cutadapt removes adapter sequences from high-throughput sequencing reads. EMBnet J. 2011;17:10-2.

30. Haas BJ, Gevers D, Earl AM, Feldgarden M, Ward DV, Giannoukos G, et al. Chimeric 16S rRNA sequence formation and detection in Sanger and 454-pyrosequenced PCR amplicons. Genome Res. 2011;21:494-504.

31. Quast C, Pruesse E, Yilmaz P, Gerken J, Schweer T, Yarza P, et al. The SILVA ribosomal RNA gene database project: improved data processing and web-based tools. Nucleic Acids Res. 2013;41:D590-596.

32. Edgar RC, Haas BJ, Clemente JC, Quince C, Knight R. UCHIME improves sensitivity and speed of chimera detection. Bioinforma. 2011;27:2194-200.

33. Edgar RC. UPARSE: highly accurate OTU sequences from microbial amplicon reads. Nat Methods. 2013;10:996-8.

34. Gevers D, Kugathasan S, Denson LA, Vázquez-Baeza Y, Treuren WV, Ren B, et al. The treatment-naive microbiome in new-onset Crohn's disease. Cell Host Microbe. 2014;15:382-92.

35. Lynch G, Guo W, Sarkar SK, Finner H. The control of the false discovery rate in fixed sequence multiple testing. Electron J Stat. 2017;11:4649-73.

36. Liaw A, Wiener M. Classification and regression by randomForest. R. N. 2002;2:18-22.

37. Breiman L. Random Forests. Machine Learn. 2001;45:5-32.

38. Milligan GW, Cooper MC. A study of standardization of variables in cluster analysis. J Classification. 1988;5:181-204.

39. Segata N, Izard J, Waldron L, Gevers D, Miropolsky L, Garrett WS, et al. Metagenomic biomarker discovery and explanation. Genome Biol. 2011;12:R60.

40. Bates D, Mächler M, Bolker B, Walker S. Fitting linear mixed-effects models using Ime4. Stat Comput. 2014;1406:133-99.

41. Vindegaard N, Speyer H, Nordentoft M, Rasmussen S, Benros ME. Gut microbial changes of patients with psychotic and affective disorders: a systematic review. Schizophr Res. 2020;S0920-9964:30584-5.

42. McBurney MI, Davis C, Fraser CM, Schneeman BO, Huttenhower C, Verbeke K, et al. Establishing what constitutes a healthy human gut microbiome: state of the science, regulatory considerations, and future directions. J Nutr. 2019;149:1882-95.

43. Kehrmann J, Menzel J, Saeedghalati M, Obeid R, Schulze C, Holzendorf V, et al. Gut microbiota in human immunodeficiency virus-infected individuals linked to coronary heart disease. J Infect Dis. 2019;219:497-508.

44. Borgo F, Macandog AD, Diviccaro S, Falvo E, Giatti S, Cavaletti G, et al. Alterations of gut microbiota composition in post-finasteride patients: a pilot study. J Eendocrinological Investig. 2020;44:1263-73.

45. Pelka-Wysiecka J, Kaczmarczyk M, Bąba-Kubiś A, Liśkiewicz P, Wroński M, Skonieczna-Żydecka K, et al. Analysis of gut microbiota and their metabolic potential in patients with schizophrenia treated with olanzapine: results from a six-week observational prospective cohort study. J Clin Med. 2019;8:1605. 
46. Maier L, Pruteanu M, Kuhn M, Zeller G, Telzerow A, Anderson EE, et al. Extensive impact of non-antibiotic drugs on human gut bacteria. Nature. 2018;555:623-8

47. Zimmermann M, Zimmermann-Kogadeeva M, Wegmann R, Goodman AL. Mapping human microbiome drug metabolism by gut bacteria and their genes. Nature. 2019;570:462-7.

48. Ghattamaneni NKR, Panchal SK, Brown L. An improved rat model for chronic inflammatory bowel disease. Pharmacol Rep. 2019;71:149-55.

49. Murphy EF, Cotter PD, Healy S, Marques TM, O'Sullivan O, Fouhy F, et al. Composition and energy harvesting capacity of the gut microbiota: relationship to diet, obesity and time in mouse models. Gut. 2010;59:1635-42.

50. Strati F, Cavalieri D, Albanese D, De Felice C, Donati C, Hayek J, et al. New evidences on the altered gut microbiota in autism spectrum disorders. Microbiome. 2017;5:24.

51. Song $X$, Lv L, Li W, Hao Y, Zhao J. The interaction of nuclear factor-kappa B and cytokines is associated with schizophrenia. Biol Psychiatry. 2009;65:481-8.

52. Song X, Fan X, Li X, Zhang W, Gao J, Zhao J, et al. Changes in pro-inflammatory cytokines and body weight during 6-month risperidone treatment in drug naïve, first-episode schizophrenia. Psychopharmacology. 2014;231:319-25.

53. Kamada N, Seo S, Chen G, Núñez G. Role of the gut microbiota in immunity and inflammatory disease. Nat Rev. Immunol. 2013;13:321-35.

54. vaedden de Steeg E, Schuren F, Obach RS, van Woudenbergh C, Walker GS, Heerikhuisen $\mathrm{M}$, et al. An ex vivo fermentation screening platform to study drug metabolism by human gut microbiota. Drug Metab Dispos. 2018;46:1596-607.

55. Liu G, Chong H, Chung F, Li Y, Liong M. Lactobacillus plantarum DR7 modulated bowel movement and gut microbiota associated with dopamine and serotonin pathways in stressed adults. Int J Mol Sci. 2020;21:4608.

56. Gao H, Jiang Q, Ji H, Ning J, Li C, Zheng H. Type 1 diabetes induces cognitive dysfunction in rats associated with alterations of the gut microbiome and metabolomes in serum and hippocampus. Biochimica et. biophysica acta Mol. basis Dis. 2019;1865:165541.

57. Dinan T, Borre Y, Cryan J. Genomics of schizophrenia: time to consider the gut microbiome? Mol Psychiatry. 2014;19:1252-57.

\section{ACKNOWLEDGEMENTS}

The authors thank all the patients and their families, the healthy volunteers for their participation, and the psychiatrist who helped us take clinical data and blood samples We thank the Biological Sample Bank of The First Affiliated Hospital of Zhengzhou University for helping us store the samples. We thank the Academy of Medical Science, Zhengzhou University for providing us with a perfect experimental platform. This study was funded by the National Natural Science Foundation of China (No. 81971253 to X-OS), Zhong yuan Innovation Leading Talents of the Thousand Talents Plan
(204200510019), Medical science and technology foundation of health and family planning commission of Henan province (SBGJ201808 to X-QS), Project for Science and Technology Innovation Teams in Universities of Henan Province (21IRTSTHN027). School and Hospital Co-incubation Funds of Zhengzhou University (No. 2017-BSTDJJ-04 to X-QS), and UiO: Lifesciences Convergence environment, University of Oslo, Norway (project 4MENT to YW, OAA), and Research Council of Norway (\#223273; \#302854).

\section{COMPETING INTERESTS}

OAA is a consultant to HealthLytix and has received speaker's honorarium from Lundbreck and Sunovion. The other authors declare no conflicts of interest.

\section{ADDITIONAL INFORMATION}

Supplementary information The online version contains supplementary material available at https://doi.org/10.1038/s41398-021-01531-3.

Correspondence and requests for materials should be addressed to S.H. or X.S.

Reprints and permission information is available at http://www.nature.com/ reprints

Publisher's note Springer Nature remains neutral with regard to jurisdictional claims in published maps and institutional affiliations.

(i) Open Access This article is licensed under a Creative Commons Attribution 4.0 International License, which permits use, sharing, adaptation, distribution and reproduction in any medium or format, as long as you give appropriate credit to the original author(s) and the source, provide a link to the Creative Commons license, and indicate if changes were made. The images or other third party material in this article are included in the article's Creative Commons license, unless indicated otherwise in a credit line to the material. If material is not included in the article's Creative Commons license and your intended use is not permitted by statutory regulation or exceeds the permitted use, you will need to obtain permission directly from the copyright holder. To view a copy of this license, visit http://creativecommons. org/licenses/by/4.0/.

(c) The Author(s) 2021 